Check for updates

Cite this: Mater. Chem. Front. 2020, 4, 941

Received 9th December 2019 Accepted 21st January 2020

DOI: 10.1039/c9qm00744j

rsc.li/frontiers-materials

\section{Achieving remarkable and reversible mechanochromism from a bright ionic AlEgen with high specificity for mitochondrial imaging and secondary aggregation emission enhancement for long-term tracking of tumors $\dagger$}

\author{
Xinzhe Yang, † $^{\mathrm{a}}$ Qian Wang, $\ddagger^{\mathrm{bc}}$ Peiyu $\mathrm{Hu}^{\mathrm{b}}{ }^{\mathrm{b}}$ Chao Xu, ${ }^{a}$ Wenjing Guo, ${ }^{\mathrm{b}}$ Zhi Wang, \\ Zhu Mao, (D) ${ }^{d}$ Zhan Yang, ${ }^{d}$ Cong Liu, ${ }^{a}$ Guang Shi, ${ }^{* a}$ Ling Chen, ${ }^{\text {b }}$ Bingjia Xu (D) *ae \\ and Zhenguo $\mathrm{Chi}$ (D) ed
}

\begin{abstract}
Strong intermolecular interactions that are in favour of elevating the luminescence efficiencies of aggregation-induced emission luminogens (AlEgens) are usually detrimental to the generation of obvious mechanochromism (MC). Therefore, it remains a challenge to develop MC-active AIE compounds that show high fluorescence quantum yield and a significant emission wavelength change simultaneously. Herein, a purely organic AlEgen and its ionic species, namely, TCPy and TCPyP, have been constructed by simultaneously employing tetraphenylethene (TPE) and phenylacrylonitrile as essential chromophores. It is found that both luminogens are highly emissive in the solid state and afford content $\Phi_{\mathrm{F}, \mathrm{S}}$ values of 0.90 and 0.52, respectively. Concurrently, TCPy has been determined to possess mechanochromism (MC) properties, but only gives a small emission variation of $14 \mathrm{~nm}$ under mechanical stimuli. By contrast, TCPyP exhibits remarkable and reversible $M C$ with a large $\Delta \lambda_{\mathrm{em} \text { max }}$ value of up to $90 \mathrm{~nm}$, representing the state-of-the-art MC performance for ionic AlEgens. Single crystal analysis reveals that the prominent MC of TCPyP mainly originates from the presence of bulky hexafluorophosphate anions in the crystal structure, which can enlarge the intermolecular distance to slightly weaken the intermolecular interactions and thereby result in the transformation of $\mathrm{H}$-aggregates to $\mathrm{J}$-aggregates upon grinding treatment. Besides, TCPyP shows much higher mitochondrial specificity in comparison with TCPy and enables mitochondrial staining with high brightness and good biocompatibility in living cells. Moreover, an abnormal phenomenon of secondary aggregation emission enhancement is observed by using this ionic AlEgen as a bioprobe for in vivo imaging, which allows long-term tracking of the tumor in nude mice. All these results suggest that the ionic AlEgen TCPyP has potential for various practical applications, including sensing, anti-counterfeiting, cell imaging and cancer diagnosis.
\end{abstract}

\footnotetext{
${ }^{a}$ Key Laboratory of Theoretical Chemistry of Environment, Ministry of Education, Guangzhou Key Laboratory of Analytical Chemistry for Biomedicine, School of Chemistry, South China Normal University, Guangzhou 510006, China. E-mail: bingiiaxu@m.scnu.edu.cn, shiguang@scnu.edu.cn

${ }^{b}$ State Key Lab of Respiratory Disease, Guangzhou Institutes of Biomedicine and Health, Chinese Academy of Sciences, Guangzhou, China.

E-mail: chen_ling@gibh.ac.cn

${ }^{c}$ University of Chinese Academy of Sciences, Beijing, China

${ }^{d}$ School of Chemistry, Sun Yat-sen University, Guangzhou 510275, China

${ }^{e}$ State Key Laboratory of Optoelectronic Materials and Technologies

(Sun Yat-sen University), Guangzhou 510006, China

$\dagger$ Electronic supplementary information (ESI) available: Details of the synthesis, structural information for the compounds (NMR and mass spectra), Fig. S1-S28 and Tables S1-S3. CCDC 1948629 and 1948630. For ESI and crystallographic data in CIF or other electronic format see DOI: 10.1039/c9qm00744j

\$ These authors contributed equally to this work.
}

\section{Introduction}

Mechanochromic emitters, which exhibit switchable luminescence in response to mechanical stimuli such as grinding, shearing or rubbing have attracted tremendous attention due to their academic importance and potential applications in optical displays, information storage, mechanical sensors, and security inks. ${ }^{1}$ In general, ideal mechanochromic materials should meet the requirements of high solid-state luminescence efficiency $\left(\Phi_{\mathrm{F}, \mathrm{S}}\right)$, notable emission variation $\left(\Delta \lambda_{\mathrm{em}, \max }\right)$ and excellent reversibility. ${ }^{2}$ Therefore, aggregation-induced emission luminogens (AIEgens) are preferable candidates in comparison with conventional organic light-emitting materials, because they can perfectly surmount the notorious aggregation-caused quenching (ACQ) effect and emit 
intense luminescence in the solid state. ${ }^{3}$ In view of this, numerous AIEgens with mechanochromic properties derived from acrylonitrile, 9,10-distyrylanthracene (DSA), tetraphenylethene (TPE), phenothiazine etc. have been sequentially created and extensively studied in the last few years. ${ }^{4}$ Concurrently, mechanisms for the mechanochromism (MC) phenomena of most AIE compounds are proposed to be associated with alterations of the molecular conformations and molecular packing modes, which are generally initiated by destruction of intermolecular interactions. ${ }^{4 e, 5}$ In this context, strong intermolecular interactions that are in favour of elevating the luminescence efficiencies of AIEgens may be detrimental to the generation of obvious MC to some extent. For example, acrylonitrile-substituted TPE derivatives, which can build plenty of robust hydrogen bonds in crystals usually afford content fluorescence quantum yields but relatively poor MC performance (Fig. S1 and Table S1, ESI $\dagger$ ). ${ }^{6}$ Consequently, MC-active AIE compounds that show high fluorescence efficiency and significant emission wavelength change simultaneously are quite limited, and molecular designs for achieving such fantastic AIE emitters are highly desirable.

Notably, ionic AIEgens have emerged as a new class of important multifunctional luminophores recently. ${ }^{3 b, 7}$ In their crystal structures, the counter anions always surround the cationic molecules because of the electrostatic interactions. ${ }^{8}$ The presence of anions may enlarge the intermolecular distance to slightly weaken the intermolecular interactions, making alterations of the molecular conformations and molecular packing modes possible under mechanical stimuli. That is, ionic AIEgens have potential to be excellent MC emitters. On the other hand, they can also make use of the negative potential gradient of energized mitochondria to accumulate selectively within the organelles and then serve as light-up fluorescent probes for bioimaging and/or efficient photosensitizers for photodynamic therapy. ${ }^{9}$ In particular, the mitochondrial membrane potentials of cancer cells are more negative than those of normal cells, which provides opportunities to enrich the cancer-cell mitochondria with more cationic AIEgens for high-quality imaging and image-guided tumor therapy. ${ }^{10}$ In consideration of their great potential as intelligent sensors as well as diagnostic and therapeutic agents, lots of ionic AIEgens with various emission maxima and different counter anions have been developed by alkylation on the position of the nitrogen or phosphorus atom. ${ }^{4 c, 7 b, 11}$ Some of them even have been identified as smart materials, displaying obvious emission variations upon force treatment. ${ }^{8 a, 12}$ However, due to the strong intramolecular charge transfer (ICT), most of these ionic AIE compounds show unsatisfactory luminescent efficiencies in the aggregation state (Fig. S2 and Table S2, ESI $\dagger$ ), ${ }^{8 b, 12 a, 12 c, 13}$ which largely confines their practical applications in optoelectronic and/or biomedical fields. In fact, ionic AIEgens can emit strong fluorescence in the solid state. ${ }^{8 a, 14}$ Therefore, notable MC and high $\Phi_{\mathrm{F}, \mathrm{s}}$ values should be achievable simultaneously on ionic AIEgens through rational optimization of their molecular structures. Although great efforts have been devoted, the development of ionic AIE emitters that can commendably satisfy the requirements of superior MC materials besides enabling high-quality bioimaging remains a formidable challenge.
In this work, we present an ionic AIEgen that shows intense luminescence together with remarkable and reversible MC in the solid state. Herein, typical AIE moieties of TPE and phenylacrylonitrile were both employed as building blocks to construct the molecule, followed by incorporation of pyridine to offer an ionization position. It is hoped that the electron-withdrawing unit of phenylacrylonitrile between the TPE and the pyridinium cation can alleviate the ICT and increase the overlap of the HOMO and LUMO to enhance the luminescence of the ionic compound. ${ }^{15}$ Thereafter, hexafluorophosphate was introduced as a counter anion to tune the molecular packing owing to its relatively large size. As a result, a purely organic AIEgen and its ionic species with satisfactory fluorescence quantum yields were achieved through this simple molecular design. In contrast to the poor MC performance of the precursor compound, conspicuous emission alteration was observed for the resulting ionic AIEgen when its pristine sample was ground. In addition, the ionic AIE emitter was found to be applicable in mitochondrial imaging with good biocompatibility and high specificity. More interestingly, it exhibited secondary aggregation emission enhancement through blood circulation, leading to long term tracking of tumors in mice. Such a smart ionic luminogen derived from TPE and phenylacrylonitrile is impressive and promising for both optoelectronic and biomedical applications.

\section{Results and discussion}

Synthetic procedures of the desired emitters, namely, TCPy and TCPyP (Fig. 1), are outlined in Scheme S1 (ESI $\dagger$ ). Briefly, the precursor compound TCPy was prepared via Knoevenagel condensation of 4-(1,2,2-triphenylvinyl)benzaldehyde and 2-(4-bromophenyl)acetonitrile in the presence of alkali, followed by a palladium-catalyzed Suzuki reaction involving the commercially available compound 4-(4,4,5,5-tetramethyl-1,3,2-dioxaborolan-2-yl)pyridine. Afterwards, methylation on the pyridine moiety and anion exchange from iodine to hexafluorophosphate were successively conducted to give the final product of TCPyP. Their chemical structures were fully confirmed by nuclear magnetic resonance (NMR) spectra and mass spectra (MS) with satisfactory results.

In dilute 1,4-dioxane solution (Fig. S3, ESI†), TCPy shows intense absorption bands at around $308 \mathrm{~nm}$ and $374 \mathrm{~nm}$, which are assigned to localized $\pi-\pi^{*}$ transition and intermolecular charge transfer (ICT) transition of the molecule, respectively. After ionization, the absorption peaks of TCPyP red shift to $316 \mathrm{~nm}$ and $387 \mathrm{~nm}$, respectively, indicating that hyperconjugation

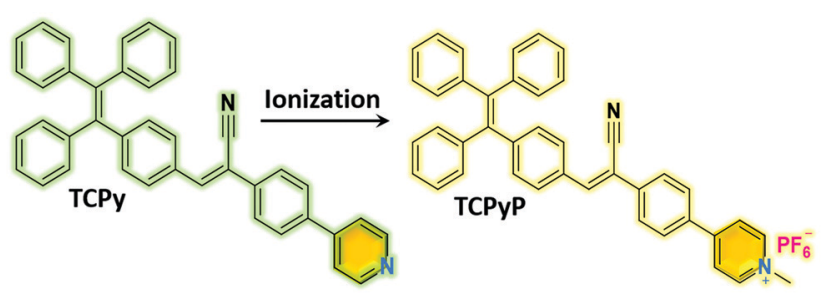

Fig. 1 Molecular structures of TCPy and TCPyP. 
probably occurs between the pyridine and methyl group and the ICT effect is enhanced in comparison with that of TCPy. ${ }^{12 a}$ As a result, the emission maximum of TCPyP $\left(\lambda_{\mathrm{em}, \max }=570 \mathrm{~nm}\right)$ becomes much redder than that of TCPy $\left(\lambda_{\mathrm{em}, \max }=516 \mathrm{~nm}\right)$ in polar 1,4-dioxane solution. The stronger ICT of the ionic compound is also confirmed by its more obvious solvatochromism, in which the emission gradually red shifts with the increase of solvent polarity (Fig. S4, ESI $\dagger$ ). Further study then focused on the possible AIE properties of these two luminogens as their multiple aromatic rings can rotate quickly to dissipate the excited state energy in the solutions and such rotational motions may be largely restricted in the aggregate state. To provide a comprehensive investigation, PL spectra of TCPy and TCPyP were measured in water/THF mixtures with different water fractions $\left(f_{\mathrm{w}}\right)$. As shown in Fig. 2, these two compounds were weakly emissive when they were well dissolved in pure THF. However, after adding a large amount of water to the solutions, significant elevations in PL intensity were observed and Mie scattering effects in UV-visible absorption were recorded (Fig. S5, ESI $\dagger$ ). Evidently, the luminescence enhancements of TCPy and TCPyP originate from the formation of nanoaggregates, manifesting that both luminogens are AIE-active. ${ }^{3 a, 16}$ When $f_{\mathrm{w}}$ increased from $95 \%$ to $99 \%$, the emission maxima of TCPy and TCPyP showed a red-shift (from $508 \mathrm{~nm}$ to $525 \mathrm{~nm}$ ) and a blueshift (from $593 \mathrm{~nm}$ to $528 \mathrm{~nm}$ ), respectively, which may be caused by the different molecular packing modes of the nanoaggregates. In addition, by prolonging the standing time, the luminescence of the nanosuspensions with $99 \%$ water content was unchanged (Fig. S6, ESI $\dagger$ ), suggesting that the molecular packing modes of their nanoaggregates are very stable. $n$-Hexane, an organic solvent with low polarity, was used instead of water to further investigate their AIE behaviours (Fig. S7, ESI $\dagger$ ). It is found that similar emission enhancement was observed for TCPyP in the mixture of THF with high $n$-hexane fraction. However, TCPy showed weak emission in the $n$-hexane/THF mixtures, because its molecules fail to aggregate. Gratifyingly, TCPy emits strong green light $\left(\lambda_{\mathrm{em}, \max }=\right.$ $512 \mathrm{~nm}$ ) with a high $\Phi_{\mathrm{s}, \mathrm{o}}$ value of up to 0.90 in the solid state (Fig. 3), which gives further strong evidence to verify its unique AIE properties. The luminescence of TCPy is identified to be prompt fluorescence and displays a rapid decay with a short lifetime of 1.95 ns (Fig. S8, ESI $\dagger$ ). Similarly, the ionic luminogen of TCPyP
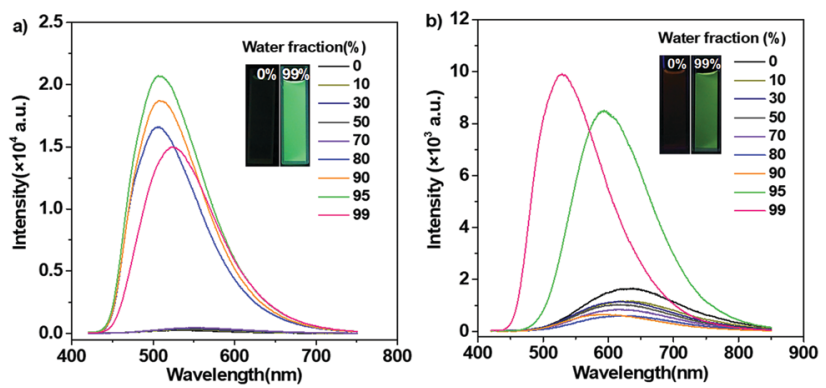

Fig. 2 PL spectra of TCPy (a) and TCPyP (b) in water/THF mixtures with different water fractions. The insets in (a) or (b) are fluorescence images of the compounds in pure THF and in water/THF mixtures with $99 \%$ water fraction under the illumination of $365 \mathrm{~nm}$ UV light, (excitation: $365 \mathrm{~nm}$; concentration: $10 \mu \mathrm{M})$.
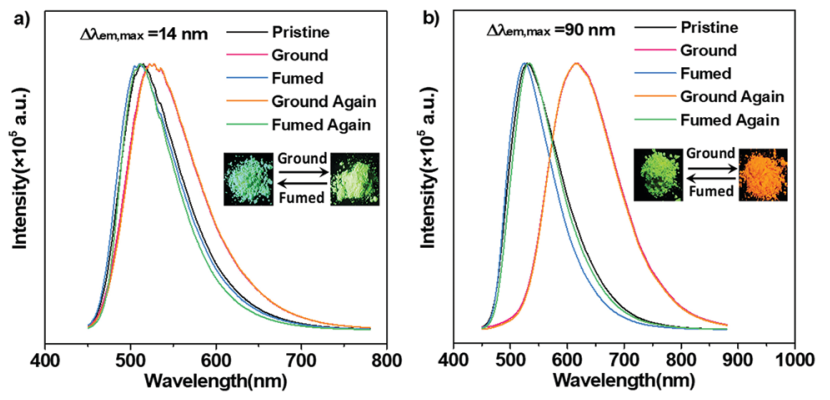

Fig. 3 Emission switching of TCPy (a) and TCPyP (b) under the stimuli of mechanical force and dichloromethane (DCM) vapour. After grinding, the emission maxima can be recovered by fuming with DCM vapour for about $10 \mathrm{~min}$. The insets in (a) and (b) are the fluorescence images of the pristine and ground samples of TCPy and TCPyP, respectively.

likewise decays rapidly, producing intense green fluorescence at $527 \mathrm{~nm}(\tau=1.05 \mathrm{~ns})$. Its $\Phi_{\mathrm{s}, \mathrm{o}}$ value was determined to be 0.52 , which is among the highest of the ionic AIEgens. ${ }^{3 b, 8 a}$ Such impressive fluorescence quantum yields of TCPy and TCPyP mainly benefit from their elaborate molecular design of simultaneously employing TPE and phenylacrylonitrile as essential chromophores.

To evaluate the MC behaviours of TCPy and TCPyP, PL measurements for their ground samples were carried out. As expected, TCPy displays poor MC performance similar to what has been observed for some other acrylonitrile-substituted TPE derivatives. Upon grinding heavily, the PL maximum of TCPy only shifts to $526 \mathrm{~nm}\left(\Phi_{\mathrm{s}, \mathrm{g}}=0.62\right)$, exhibiting a slight emission wavelength change of $14 \mathrm{~nm}$. In sharp contrast, TCPyP is highly sensitive to external force. When mild grinding treatment was applied to the pristine sample, its emission immediately became red and the corresponding maximum was established to be $617 \mathrm{~nm}\left(\Phi_{\mathrm{s}, \mathrm{g}}=0.33\right)$. Moreover, after exposure to dichloromethane vapour, the red emission of ground TCPyP disappeared and the green fluorescence rose again. That is, TCPyP shows remarkable and reversible MC with a large $\Delta \lambda_{\text {em,max }}$ value of up to $90 \mathrm{~nm}$ besides the high fluorescence quantum yield, which represents the state-of-theart MC performance for ionic AIEgens. To demonstrate its practical application, rewritable paper was made by spreading the pristine powder of TCPyP on filter paper. As depicted in Fig. S9 (ESI $\dagger$ ), the as-prepared paper exhibited strong green emission under the illumination of $365 \mathrm{~nm}$ UV light. After writing with a spatula, the letter "A" with red emission appeared, which could be readily discerned from the background owing to its high contrast in luminescence colour. When the used paper was fumed in dichloromethane vapour, the red-light-emitting letter could be erased, and the green background could be reinstalled. This study fully demonstrates that the ionic AIEgen of TCPyP has potential to be used as a recyclable anticounterfeiting or optical storage medium.

Given that the emission properties of solid emitters are closely related to their molecular packings, pristine and ground samples of TCPy and TCPyP were subjected to powder X-ray diffraction (XRD) and differential scanning calorimetry (DSC) 
analyses to understand their distinct MC behaviours. As illustrated in Fig. S10a and S11a (ESI $\dagger$ ), the sharp deflection peaks in the XRD patterns and the intense melting transitions in the DSC curves for the original powders of TCPy and TCPyP unambiguously verify that they are mainly composed of microcrystals. However, after grinding, the XRD pattern and DSC curve of TCPy are almost unchanged. Accordingly, the poor MC performance of TCPy is likely associated with its robust crystal structure that largely impedes the alteration of molecular arrangement under mechanical stimuli. On the contrary, the diffraction peaks in the XRD patterns of ground TCPyP become less defined and more diffuse (Fig. S10b, ESI $\dagger$ ). Meanwhile, exothermic transition is recorded along with the melting peak in the DSC curve (Fig. S11b, ESI $\dagger$ ). These jointly demonstrate that most of the TCPyP microcrystals are destroyed and convert to an amorphous state upon grinding, which allows the transformations of the molecular conformation and molecular packing mode to induce significant emission variation. It is worth noting that the XRD pattern and DSC curve for the fumed sample of TCPyP are almost identical to those of the pristine powder, thus indicating that its crystal structure can be completely restored to the original state with the aid of dichloromethane vapour.

Single crystal analysis and theoretical calculation based on density functional theory (DFT) were subsequently performed to gain in-depth insight into the observed strong fluorescence and MC phenomena of TCPy and TCPyP. The single crystals of these two compounds are also green-light emissive and their PL spectra are in accordance with those of the pristine powders (Fig. S12, ESI $\dagger$ ). No $\pi-\pi$ stackings were observed in the crystals with laminar structures because of their twisted molecular conformations (Fig. S13, ESI $\dagger$ ). Each unit cell of TCPy and TCPyP consists of four bulky molecules with antiparallel arrangement, suggesting their compact molecular packing modes, which are in favour of impeding the molecular motions to suppress the nonradiative decays. Moreover, the HOMO and LUMO for TCPy and the HOMO and LUMO+1 for TCPyP overlap at around the diphenylacrylonitrile fragments and show large oscillator strength with values of 0.923 and 0.539 (Fig. S14, ESI $\dagger$ ), respectively. As a result, both these two luminogens rise above the ACQ effects and emit strong fluorescence with satisfactory quantum yields in the crystalline state. As compared to TCPy, the ionic characteristic of TCPyP intensifies the ICT, thereby leading to the bathochromic shift of the emission maximum. Careful investigation reveals that numerous intermolecular interactions including two types of $\mathrm{C}-\mathrm{H} \cdots \mathrm{N}$ and four types of $\mathrm{C}-\mathrm{H} \cdots \pi$ with distances ranging from $2.535 \AA$ to $2.834 \AA$ are formed in the single crystal structure of TCPy. Their strength and distribution were simulated by Multiwfn and the results were visualized in 3D isosurfaces with different gradient colours. As presented in Fig. 4a, many regions turn into blue and green, manifesting that strong intermolecular interactions did occur. ${ }^{17}$ Therefore, the crystal structure of TCPy is very sturdy and difficult to be damaged, resulting in the small variation of the emission wavelength upon force treatment.

In the case of TCPyP, hexafluorophosphate anions insert into the interstices among the molecules and enlarge the

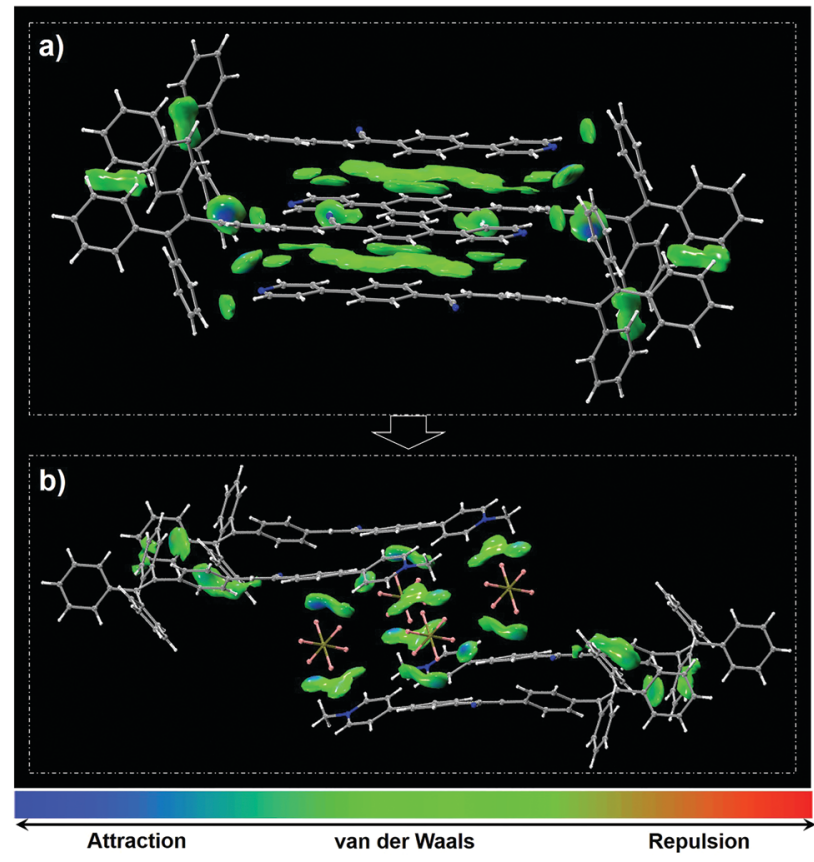

Fig. 4 Molecular packings as well as distribution and strength of intramolecular interactions in the unit cells of TCPy (a) and TCPyP (b) (isovalue $=0.5$ )

intermolecular distance from $3.033 \AA$ to $5.314 \AA$. The $\mathrm{C}-\mathrm{H} \cdots \mathrm{N}$ hydrogen bonds and most of the $\mathrm{C}-\mathrm{H} \cdots \pi$ interactions are thus replaced by $\mathrm{C}-\mathrm{H} \cdots \mathrm{F}$ (with distances ranging from $2.402 \AA$ to $2.536 \AA$ ) and P-F . . C (with distances ranging from $3.046 \AA$ to $3.066 \AA$ A interactions, which are all built by the hexafluorophosphate anions and the methylated phenylpyridine fragments. The results from the Multiwfn simulation also indicate that the intermolecular interactions mainly locate at these regions (Fig. 4b). That is, the insertion of hexafluorophosphate slightly weakened the intermolecular interactions, offering an opportunity for the generation of high contrast MC via intermolecular slippage. This viewpoint is also supported by the experimental result that the melting point of TCPyP is $8{ }^{\circ} \mathrm{C}$ lower than that of TCPy. Meanwhile, H-aggregates are found in the TCPyP crystals with green colour. Upon mild treatment of hand grinding, however, the apparent colour of the sample converts into orange and the solid-state absorption shifts from $445 \mathrm{~nm}$ to $468 \mathrm{~nm}$ (Fig. S15, ESI $\dagger$ ). These experimental data imply that mechanical stimulus probably gives rise to interlayer slippage of the crystals and induces the formation of J-aggregates, which can allow the electronic transitions between the lower excited state and ground state. ${ }^{2 a, 18}$ Also, it can be speculated that the head-to-tail packing characteristic of the J-aggregates may strengthen the dipole-dipole interactions and then enhance the ICT process of the molecules to further narrow the energy gap. Consequently, the prominent MC performance of TCPyP likely stems from the transformation of $\mathrm{H}$-aggregates to J-aggregates, which is enabled by the molecular ionization and the insertion of bulky anions. ${ }^{2 a, 19}$

Inspired by the AIE characteristics with high fluorescence quantum yields in the aggregation state, in vitro bioimaging 

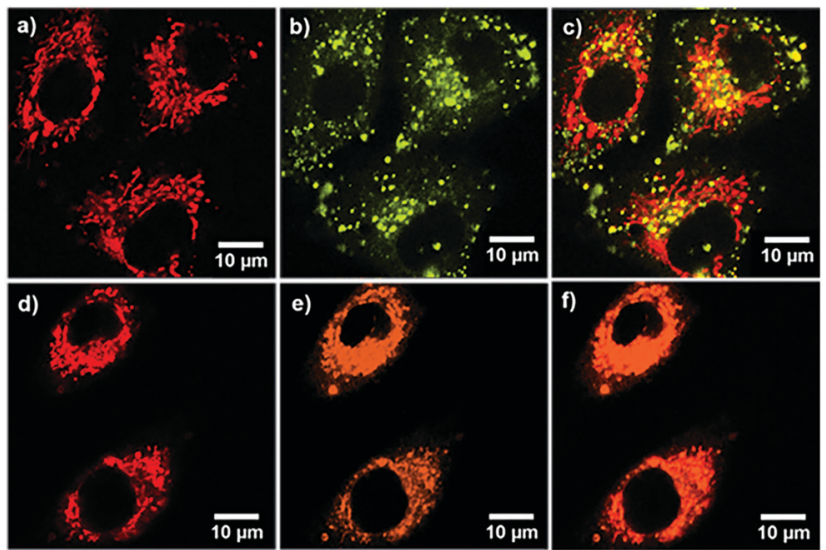

Fig. 5 CLSM images indicate the colocalization of MitoTracker Deep Red FM with TCPy $(a-c)$ and TCPyP $(d-f)$ in A549 cancer cells, respectively. ( $a$ and d) Fluorescence images of mitochondria stained by MitoTracker Deep Red FM (red pseudocolor). (b) Single-photon excited fluorescence image of mitochondria stained by TCPy. (c) An overlay image of parts (a) and (b). (e) Single-photon excited fluorescence image of mitochondria stained by TCPyP. (f) An overlay image of parts (d) and (e) (excitation: $405 \mathrm{~nm}$; concentration: $5 \mu \mathrm{M}$ ).

was carried out to demonstrate the possibilities of TCPy and TCPyP for biological applications. A CCK-8 assay on A549 cancer cells was firstly conducted to evaluate their biocompatibility. The study of dose-dependent cytotoxicity deciphers that no obvious toxicity was observed even when the cells were treated with TCPy or TCPyP at the concentration of up to $20 \mu \mathrm{M}$ (Fig. S16, ESI $\dagger$ ), suggesting that they are biocompatible. The A549 cancer cells were subsequently incubated with $5 \mu \mathrm{M}$ of TCPy or TCPyP for $2 \mathrm{~h}$. As shown in Fig. 5, it is found that both these two AIEgens successfully penetrate the cytomembranes and give bright green and orange fluorescence in the cells, respectively. Noteworthily, TCPyP can clearly visualize the reticulum-like mitochondria with excellent image contrast to the cell background. A colocalization experiment was then implemented by co-staining with MitoTracker Green (MTG), which is a commercially available indicator for mitochondria. The intense orange emission of TCPyP, which is generated by visible light excitation at $405 \mathrm{~nm}$ well overlaps the pseudo red emission of MTG with a satisfactory Pearson's coefficient $(r=0.79)$, undoubtedly demonstrating its high specificity for mitochondria-staining in living cells. By contrast, the small Pearson's coefficient ( $r=0.33$ ) for the colocalization of TCPy and MTG suggests that the TCPy molecules mainly locate outside the energy-supply organelles. These experimental phenomena fully illustrate that TCPyP exhibits much better selectivity for mitochondria in A549 cancer cells as compared to TCPy. This is reasonable because positively charged dyes like TCPyP prefer to stain mitochondria owing to their high negative membrane potential. ${ }^{10 a}$ Intriguingly, when twophoton excitation at $800 \mathrm{~nm}$ was applied for cell imaging (Fig. S17, ESI $\dagger$ ), TCPyP also shows large overlap with MTG (Pearson's coefficient of 0.75). That is, this ionic AIEgen can likewise serve as a two-photon excitation bioprobe, which has potential for practical applications in three-dimensional fluorescence imaging with weak photodamage, deep tissue penetration and low fluorescence background. ${ }^{20}$ Although the two-photon absorption cross-section $\left(\sigma_{2}=5.7 \mathrm{GM}\right)$ of TCPyP is relatively small (Fig. S18, ESI $\dagger$ ), it can still give clear images of mitochondria, which may benefit from its high fluorescence quantum yield.

\section{a)}

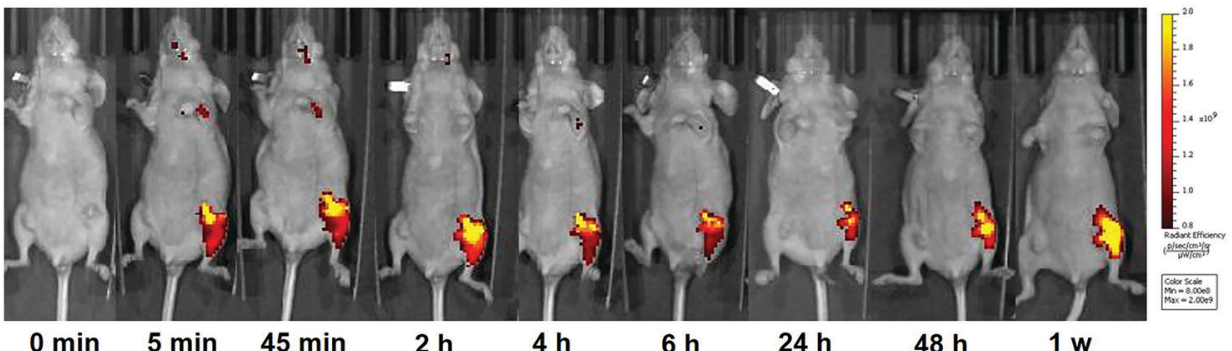

b)
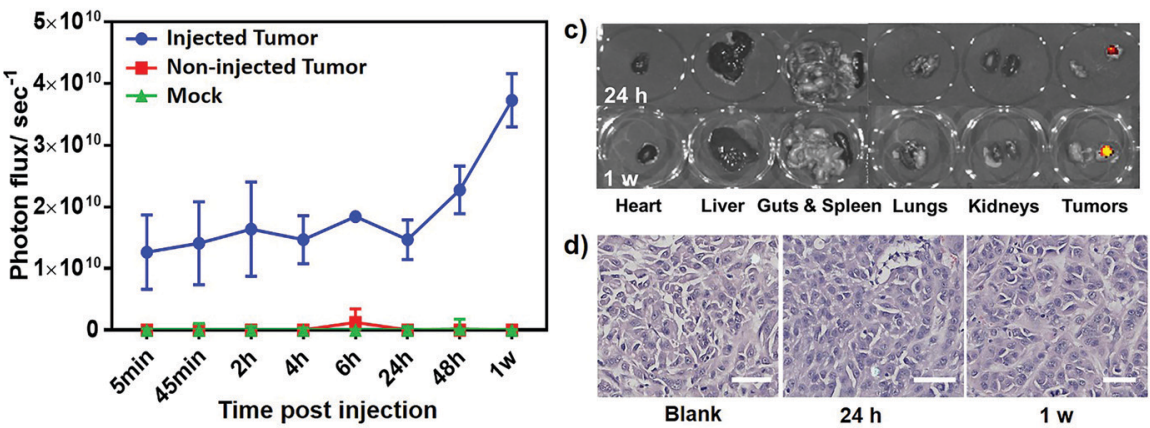

Fig. 6 In vivo imaging. (a) Biodistribution of TCPyP in an A549-tumor-bearing mouse after intratumoral injection of TCPyP (10 mM, $100 \mu \mathrm{L})$ at different times. (b) Luminescence intensity recorded from the injected and non-injected tumors of the mice at different times. (c) Ex vivo fluorescence images of tumor tissues and some organs at different times from the mice injected with TCPyP. The mice were sacrificed $24 \mathrm{~h}$ and 7 days after injection of the ionic AlEgen. (d) H\&E staining of the tumor tissues from mice intratumorally injected with or without TCPyP. Scale bar: $50 \mu \mathrm{m}$. 
In consideration of its prominent performance in cellular experiments, TCPyP was selected as a bioprobe for in vivo imaging involving athymic nude mice. A tumor model was implanted subcutaneously in each posterior flank of the mice, and the ionic AIEgen was injected to the one on the right side. As depicted in Fig. 6a, fluorescence from the tumor became obvious after intratumoral injection of TCPyP for $5 \mathrm{~min}$ and reached a maximum at about $2 \mathrm{~h}$. The continuous increase of emission intensity at this stage is probably caused by aggregation of the AIE-active TCPyP molecules, which can intensify their luminescence through restriction of molecular motions. The light-up imaging process and the intense fluorescence observed at the tumor site collectively verify that TCPyP can likewise surmount the ACQ effect in biological systems and render high quality tumor imaging in living mice. Weak fluorescence signals were also recorded at the head and heart positions, suggesting that some of the ionic AIE molecules entered the blood circulation and then tentatively accumulated at the brain and blood-supply organs. Although more TCPyP diffused to the circulation system for the duration from $2 \mathrm{~h}$ to $24 \mathrm{~h}$, the tumor was still clearly and continuously imaged by the residual molecules. This positive result should be assigned to their excellent luminescence performance, which is helpful for medical diagnosis.

Unexpectedly, a more conspicuous light-up process was observed at the injected tumor site when the imaging time was further extended. The fluorescence intensity monitored at 1 week postinjection was even up to twice that at $2 \mathrm{~h}$ (Fig. 6b), manifesting an abnormal phenomenon of secondary aggregation emission enhancement (SAEE). In other words, the unmetabolized TCPyP molecules in the circulation system can accumulate again at the tumor through blood circulation, which may be ascribed to the enhanced permeability and retention (EPR) effect of neoplastic tissues as well as the unique molecular structure and the ionic characteristic of TCPyP. ${ }^{21}$ Thus, SAEE occurred from $24 \mathrm{~h}$ to 7 days, leading to long-term tracking of the tumor. By contrast, only an extremely weak fluorescence signal could be detected from the non-injected tumor model. This result may be caused by its relatively low TCPyP concentration, which cannot reach the threshold of aggregation to activate the intrinsic AIE property of the molecules. To further explore the origin of the observed sAEE phenomenon, the mice were sacrificed after injection of the ionic AIEgen for $24 \mathrm{~h}$ and 7 days, and ex vivo fluorescence images of isolated tumors and some organs are shown in Fig. 6c. It is found that the luminescent molecules mainly accumulated in the injected neoplastic tissues giving high emission intensity. In sharp contrast, no obvious fluorescence signals were detected from the major organs, including the heart, liver, spleen, lungs, kidneys, and intestines. Moreover, the luminescence of the tumor at 7 days was much stronger than that at $24 \mathrm{~h}$. On the other hand, an intravenous injection experiment was performed on nude mice and the ex vivo fluorescence images of their isolated tumors were achieved. The luminescence on neoplastic tissues gradually enhanced with the increase of blood circulation time (Fig. S19, ESI $\dagger$ ), clearly demonstrating that TCPyP also can accumulate at the tumor site by intravenous injection. Obviously, the EPR effect of neoplastic tissues and the unique molecular structure of TCPyP played important roles in the SAEE process. Hematoxylin and eosin (H\&E) staining for histological analysis was subsequently performed to evaluate the cytotoxicity of TCPyP to live mice at $24 \mathrm{~h}$ and 1 week postinjection. For the experimental group, no noticeable inflammation, cell death or apoptosis was found in the injected tumors of mice (Fig. 6d), indicating the high biocompatibility of the compound. All these results fully demonstrate that TCPyP holds promising potential for in vivo bioimaging.

\section{Conclusions}

In summary, a purely organic AIEgen and its ionic species, namely, TCPy and TCPyP, which are highly emissive in the solid state have been developed by simultaneously employing TPE and phenylacrylonitrile as essential chromophores. It is found that TCPyP shows remarkable and reversible MC, which is distinctively different from that of its precursor compound. The impressive MC phenomenon of the ionic AIEgen has been identified to originate from the presence of bulky hexafluorophosphate anions in the crystal structure, which slightly weakens the intermolecular interactions and thereby results in the transformation of $\mathrm{H}$-aggregates to J-aggregates under mechanical stimuli. Benefiting from the lipophilic and cationic molecular structure with two typical AIE units, TCPyP also exhibits high brightness and specificity as well as good biocompatibility for mitochondria-staining in living cells. Furthermore, secondary aggregation emission enhancement is observed by using this ionic AIEgen as a biprobe for in vivo imaging, allowing long-term tracking of tumors in nude mice. All these intriguing results suggest that TCPyP has the potential for innovative applications in sensors, security systems and cancer diagnosis.

\section{Conflicts of interest}

There are no conflicts to declare.

\section{Acknowledgements}

This work was supported by the National Natural Science Foundation of China (51603233), the Guangzhou Health Care and Cooperative Innovation Major Project (201604020011), the Natural Science Foundation of Guangdong Province of China (2019A1515010550), the Pearl River S\&T Nova Program of Guangzhou (201806010125), the Opening Foundation of State Key Laboratory of Optoelectronic Materials and Technologies (Sun Yat-sen University, OEMT-2018-KF-11) and the Research Start-up Funds for Outstanding Young Scholars of South China Normal University (8S0112).

\section{Notes and references}

1 (a) Z. Chi, X. Zhang, B. Xu, X. Zhou, C. Ma, Y. Zhang, S. Liu and J. Xu, Recent advances in organic mechanofluorochromic 
materials, Chem. Soc. Rev., 2012, 41, 3878-3896; (b) Y. Sagara and T. Kato, Mechanically induced luminescence changes in molecular assemblies, Nat. Chem., 2009, 1, 605-610; (c) Z. Mao, Z. Yang, Y. Mu, Y. Zhang, Y. F. Wang, Z. Chi, C. C. Lo, S. Liu, A. Lien and J. Xu, Linearly tunable emission colors obtained from a fluorescent-phosphorescent dualemission compound by mechanical stimuli, Angew. Chem., Int. Ed., 2015, 54, 6270-6273; (d) Z. Ma, M. Teng, Z. Wang, S. Yang and X. Jia, Mechanically induced multicolor switching based on a single organic molecule, Angew. Chem., Int. Ed., 2013, 52, 12268-12272; (e) Y. Sagara and T. Kato, Brightly tricolored mechanochromic luminescence from a singleluminophore liquid crystal: reversible writing and erasing of images, Angew. Chem., Int. Ed., 2011, 50, 9128-9132; $(f)$ B. Xu, H. Wu, J. Chen, Z. Yang, Z. Yang, Y. C. Wu, Y. Zhang, C. Jin, P. Y. Lu, Z. Chi, S. Liu, J. Xu and M. Aldred, White-light emission from a single heavy atom-free molecule with room temperature phosphorescence, mechanochromism and thermochromism, Chem. Sci., 2017, 8, 1909-1914; (g) S. K. Park, I. Cho, J. Gierschner, J. H. Kim, J. H. Kim, J. E. Kwon, O. K. Kwon, D. R. Whang, J. H. Park, B. K. An and S. Y. Park, Stimuliresponsive reversible fluorescence switching in a crystalline donor-acceptor mixture film: mixed stack charge-transfer emission versus segregated stack monomer emission, Angew. Chem., Int. Ed., 2016, 55, 203-207.

2 (a) S. J. Yoon, J. W. Chung, J. Gierschner, K. S. Kim, M. G. Choi, D. Kim and S. Y. Park, Multistimuli two-color luminescence switching via different slip-stacking of highly fluorescent molecular sheets, J. Am. Chem. Soc., 2010, 132, 13675-13683; (b) X. Luo, J. Li, C. Li, L. Heng, Y. Q. Dong, Z. Liu, Z. Bo and B. Z. Tang, Reversible switching of the emission of diphenyldibenzofulvenes by thermal and mechanical stimuli, Adv. Mater., 2011, 23, 3261-3265; (c) W. Z. Yuan, Y. Tan, Y. Gong, P. Lu, J. W. Lam, X. Y. Shen, C. Feng, H. H. Sung, Y. Lu, I. D. Williams, J. Z. Sun, Y. Zhang and B. Z. Tang, Synergy between twisted conformation and effective intermolecular interactions: strategy for efficient mechanochromic luminogens with high contrast, Adv. Mater., 2013, 25, 2837-2843.

3 (a) Y. Hong, J. W. Lam and B. Z. Tang, Aggregation-induced emission: phenomenon, mechanism and applications, Chem. Commun., 2009, 4332-4353; (b) J. Mei, N. L. Leung, R. T. Kwok, J. W. Lam and B. Z. Tang, Aggregation-induced emission: together we shine, united we soar!, Chem. Rev., 2015, 115, 11718-11940; (c) J. A. Li, J. Zhou, Z. Mao, Z. Xie, Z. Yang, B. Xu, C. Liu, X. Chen, D. Ren, H. Pan, G. Shi, Y. Zhang and Z. Chi, Transient and persistent roomtemperature mechanoluminescence from a white-lightemitting AIEgen with tricolor emission switching triggered by light, Angew. Chem., Int. Ed., 2018, 57, 6449-6453.

4 (a) J. Zhao, Z. Chi, Y. Zhang, Z. Mao, Z. Yang, E. Ubba and Z. Chi, Recent progress in the mechanofluorochromism of cyanoethylene derivatives with aggregation-induced emission, J. Mater. Chem. C, 2018, 6, 6327-6353; (b) J. Zhao, Z. Chi, Z. Yang, Z. Mao, Y. Zhang, E. Ubbaa and Z. Chi, Recent progress in the mechanofluorochromism of distyrylanthracene derivatives with aggregation-induced emission, Mater. Chem. Front., 2018, 2, 1595-1608; (c) Z. Yang, Z. Chi, Z. Mao, Y. Zhang, S. Liu, J. Zhao, M. P. Aldred and Z. Chi, Recent advances in mechano-responsive luminescence of tetraphenylethylene derivatives with aggregation-induced emission properties, Mater. Chem. Front., 2018, 2, 861-890; (d) Z. Xie, Q. Huang, T. Yu, L. Wang, Z. Mao, W. Li, Z. Yang, Y. Zhang, S. Liu, J. Xu, Z. Chi and M. P. Aldred, Hydrogen-bonding-assisted intermolecular charge transfer: a new strategy to design single-component white-light-emitting materials, Adv. Funct. Mater., 2017, 27, 1703918; (e) Y. Dong, B. Xu, J. Zhang, X. Tan, L. Wang, J. Chen, H. Lv, S. Wen, B. Li, L. Ye, B. Zou and W. Tian, Piezochromic luminescence based on the molecular aggregation of 9,10-bis((E)-2-(pyrid-2-yl)vinyl)anthracene, Angew. Chem., Int. Ed., 2012, 51, 10782-10785; $(f)$ M. Okazaki, Y. Takeda, P. Data, P. Pander, H. Higginbotham, A. P. Monkman and S. Minakata, Thermally activated delayed fluorescent phenothiazine-dibenzo[ $[a, j]$ phenazine-phenothiazine triads exhibiting tricolor-changing mechanochromic luminescence, Chem. Sci., 2017, 8, 2677-2686.

5 (a) Y. Gong, G. Chen, Q. Peng, W. Z. Yuan, Y. Xie, S. Li, Y. Zhang and B. Z. Tang, Achieving persistent room temperature phosphorescence and remarkable mechanochromism from pure organic luminogens, Adv. Mater., 2015, 27, 6195-6201; (b) Y. Chen, C. Xu, B. Xu, Z. Mao, J. Li, Z. Yang, N. R. Peethani, C. Liu, G. Shi, F. L. Gu, Y. Zhang and Z. Chi, Chirality-Activated Mechanoluminescence from aggregationinduced emission enantiomers with high contrast mechanochromism and force-induced delayed fluorescence, Mater. Chem. Front., 2019, 3, 1800-1806; (c) M. Tanioka, S. Kamino, A. Muranaka, Y. Ooyama, H. Ota, Y. Shirasaki, J. Horigome, M. Ueda, M. Uchiyama, D. Sawada and S. Enomoto, Reversible near-infrared/blue mechanofluorochromism of aminobenzopyranoxanthene, J. Am. Chem. Soc., 2015, 137, 6436-6439; (d) J. Yang, J. Qin, P. Geng, J. Wang, M. Fang and Z. Li, Molecular conformation-dependent mechanoluminescence: same mechanical stimulus but different emissive color over time, Angew. Chem., Int. Ed., 2018, 57, 14174-14178.

6 (a) C. Ma, J. He, B. Xu, G. Xie, Z. Xie, Z. Mao and Z. Chi, A TPE-benzothiazole piezochromic and acidichromic molecular switch with high solid state luminescent efficiency, RSC Adv., 2018, 8, 6252-6258; (b) S. Gundu, M. Kim, N. Mergu and Y.-A. Son, AIE-active and reversible mechanochromic tetraphenylethene-tetradiphenylacrylonitrile hybrid luminogens with re-writable optical data storage application, Dyes Pigm., 2017, 146, 7-13; (c) B. Xu, M. Xie, J. He, B. Xu, Z. Chi, W. Tian, L. Jiang, F. Zhao, S. Liu, Y. Zhang, Z. Xu and J. Xu, An aggregation-induced emission luminophore with multi-stimuli single- and two-photon fluorescence switching and large two-photon absorption cross section, Chem. Commun., 2013, 49, 273-275; (d) X. Y. Shen, Y. J. Wang, E. Zhao, W. Z. Yuan, Y. Liu, P. Lu, A. Qin, Y. Ma, J. Z. Sun and B. Z. Tang, Effects of substitution with donor-acceptor groups on the properties of tetraphenylethene trimer: aggregationinduced emission, solvatochromism, and mechanochromism, J. Phys. Chem. C, 2013, 117, 7334-7347. 
7 (a) D. Ding, K. Li, B. Liu and B. Z. Tang, Bioprobes based on AIE fluorogens, Acc. Chem. Res., 2013, 46, 2441-2453; (b) S. Chen, Y. Hong, Y. Liu, J. Liu, C. W. Leung, M. Li, R. T. Kwok, E. Zhao, J. W. Lam, Y. Yu and B. Z. Tang, Fullrange intracellular $\mathrm{pH}$ sensing by an aggregation-induced emission-active two-channel ratiometric fluorogen, J. Am. Chem. Soc., 2013, 135, 4926-4929.

8 (a) M. Jiang, X. Gu, R. T. K. Kwok, Y. Li, H. H. Y. Sung, X. Zheng, Y. Zhang, J. W. Y. Lam, I. D. Williams, X. Huang, K. S. Wong and B. Z. Tang, Multifunctional AIEgens: ready synthesis, tunable emission, mechanochromism, mitochondrial, and bacterial imaging, Adv. Funct. Mater., 2018, 28, 1704589; (b) X. Gu, E. Zhao, T. Zhao, M. Kang, C. Gui, J. W. Lam, S. Du, M. M. Loy and B. Z. Tang, A mitochondrionspecific photoactivatable fluorescence turn-on AIE-based bioprobe for localization super-resolution microscope, Adv. Mater., 2016, 28, 5064-5071.

9 (a) J. Qian and B. Z. Tang, AIE luminogens for bioimaging and theranostics: from organelles to animals, Chem, 2017, 3, 56-91; (b) J. Mei, Y. Huang and H. Tian, Progress and trends in AIE-based bioprobes: a brief overview, ACS Appl. Mater. Interfaces, 2018, 10, 12217-12261; (c) X. Cai, F. Hu, G. Feng, R. T. K. Kwok, B. Liu and B. Z. Tang, Organic mitoprobes based on fluorogens with aggregation-induced emission, Isr. J. Chem., 2018, 58, 860-873.

10 (a) D. Wang, M. M. S. Lee, G. Shan, R. T. K. Kwok, J. W. Y. Lam, H. Su, Y. Cai and B. Z. Tang, Highly efficient photosensitizers with far-red/near-infrared aggregationinduced emission for in vitro and in vivo cancer theranostics, Adv. Mater., 2018, 30, e1802105; (b) X. Li, M. Jiang, J. W. Y. Lam, B. Z. Tang and J. Y. Qu, Mitochondrial imaging with combined fluorescence and stimulated Raman scattering microscopy using a probe of the aggregation-induced emission characteristic, J. Am. Chem. Soc., 2017, 139, 17022-17030; (c) Q. Hu, M. Gao, G. Feng and B. Liu, Mitochondria-targeted cancer therapy using a light-up probe with aggregation-inducedemission characteristics, Angew. Chem., Int. Ed., 2014, 53, 14225-14229.

11 (a) H. Li, J. Chang, T. Hou and F. Li, Aggregation induced emission amphiphile with an ultra low critical micelle concentration: fabrication, self assembling, and cell imaging, J. Mater. Chem. B, 2016, 4, 198-201; (b) H. Li, C. Wang, T. Hou and F. Li, Amphiphile-mediated ultrasmall aggregation induced emission dots for ultrasensitive fluorescence biosensing, Anal. Chem., 2017, 89, 9100-9107.

12 (a) N. Zhao, Z. Yang, J. W. Lam, H. H. Sung, N. Xie, S. Chen, H. Su, M. Gao, I. D. Williams, K. S. Wong and B. Z. Tang, Benzothiazolium-functionalized tetraphenylethene: an AIE luminogen with tunable solid-state emission, Chem. Commun., 2012, 48, 8637-8639; (b) N. Zhao, M. Li, Y. Yan, J. W. Y. Lam, Y. L. Zhang, Y. S. Zhao, K. S. Wong and B. Z. Tang, A tetraphenylethene-substituted pyridinium salt with multiple functionalities: synthesis, stimuli-responsive emission, optical waveguide and specific mitochondrion imaging, J. Mater. Chem. C, 2013, 1, 4640-4646; (c) Y. Wang, I. Zhang, B. Yu, X. Fang, X. Su, Y.-M. Zhang, T. Zhang, B. Yang, M. Li and
S. X.-A. Zhang, Full-color tunable mechanofluorochromism and excitation-dependent emissions of single-arm extended tetraphenylethylenes, J. Mater. Chem. C, 2015, 3, 12328-12334.

13 (a) S. Weng, Z. Si, Y. Zhou, Q. Zuo, L. Shi and Q. Duan, Derivatives of 1-benzyl-4-(4-triphenylvinylphenyl)pyridinium bromide: Synthesis, characterization, mechanofluorochromism/ aggregation-induced emission (AIE) character and theoretical simulations, J. Lumin., 2018, 195, 14-23; (b) C. Gui, E. Zhao, R. T. K. Kwok, A. C. S. Leung, J. W. Y. Lam, M. Jiang, H. Deng, Y. Cai, W. Zhang, H. Su and B. Z. Tang, AIE-active theranostic system: selective staining and killing of cancer cells, Chem. Sci., 2017, 8, 1822-1830; (c) N. Zhao, S. Chen, Y. Hong and B. Z. Tang, A red emitting mitochondria-targeted AIE probe as an indicator for membrane potential and mouse sperm activity, Chem. Commun., 2015, 51, 13599-13602; (d) F. Hu, X. Cai, P. N. Manghnani, Kenry, W. Wu and B. Liu, Multicolor monitoring of cellular organelles by single wavelength excitation to visualize the mitophagy process, Chem. Sci., 2018, 9, 2756-2761.

14 (a) T. Hu, B. Yao, X. Chen, W. Li, Z. Song, A. Qin, J. Z. Sun and B. Z. Tang, Effect of ionic interaction on the mechanochromic properties of pyridinium modified tetraphenylethene, Chem. Commun., 2015, 51, 8849-8852; (b) Z. Wang, Y. Gu, J. Liu, X. Cheng, J. Z. Sun, A. Qin and B. Z. Tang, A novel pyridinium modified tetraphenylethene: AIEactivity, mechanochromism, DNA detection and mitochondrial imaging, J. Mater. Chem. B, 2018, 6, 1279-1285.

15 (a) M. Kim, S. K. Jeon, S. H. Hwang and J. Y. Lee, Stable blue thermally activated delayed fluorescent organic lightemitting diodes with three times longer lifetime than phosphorescent organic light-emitting diodes, Adv. Mater., 2015, 27, 2515-2520; (b) W. Zeng, H. Y. Lai, W. K. Lee, M. Jiao, Y. J. Shiu, C. Zhong, S. Gong, T. Zhou, G. Xie, M. Sarma, K. T. Wong, C. C. Wu and C. Yang, Achieving nearly $30 \%$ external quantum efficiency for orange-red organic light emitting diodes by employing thermally activated delayed fluorescence emitters composed of 1,8-naphthalimideacridine hybrids, Adv. Mater., 2018, 30, 201704961; (c) L. S. Cui, H. Nomura, Y. Geng, J. U. Kim, H. Nakanotani and C. Adachi, Controlling singlet-triplet energy splitting for deep-blue thermally activated delayed fluorescence emitters, Angew. Chem., Int. Ed., 2017, 56, 1571-1575.

16 B. Xu, J. He, Y. Mu, Q. Zhu, S. Wu, Y. Wang, Y. Zhang, C. Jin, C. Lo, Z. Chi, A. Lien, S. Liu and J. Xu, Very bright mechanoluminescence and remarkable mechanochromism using a tetraphenylethene derivative with aggregation-induced emission, Chem. Sci., 2015, 6, 3236-3241.

17 (a) T. Lu and F. Chen, Multiwfn: a multifunctional wavefunction analyzer, J. Comput. Chem., 2012, 33, 580-592; (b) C. Lefebvre, G. Rubez, H. Khartabil, J. C. Boisson, J. Contreras-Garcia and E. Henon, Accurately extracting the signature of intermolecular interactions present in the NCI plot of the reduced density gradient versus electron density, Phys. Chem. Chem. Phys., 2017, 19, 17928-17936; (c) C. Lefebvre, H. Khartabil, J. C. Boisson, J. ContrerasGarcia, J. P. Piquemal and E. Henon, The independent 
gradient model: a new approach for probing strong and weak interactions in molecules from wave function calculations, ChemPhysChem, 2018, 19, 724-735.

18 P. Xue, T. Zhang and Y. Han, Multicolour- and high-colourcontrast switching in response to force and acid vapour by introducing an asymmetric $\mathrm{D}-\pi-\mathrm{A}-\pi-\mathrm{D}$ structure, J. Mater. Chem. C, 2019, 7, 9537-9544.

19 (a) W. Yuan, X. K. Ren, M. Li, H. Guo, Y. Han, M. Wu, Q. Wang, M. Li and Y. Chen, From S,N-heteroacene to large discotic polycyclic aromatic hydrocarbons (PAHs): liquid crystal versus plastic crystalline materials with tunable mechanochromic fluorescence, Angew. Chem., Int. Ed., 2018, 57, 6161-6165; (b) X. Sun, X. Zhang, X. Li, S. Liu and G. Zhang, A mechanistic investigation of mechanochromic luminescent organoboron materials, J. Mater. Chem., 2012, 22, 17332-17339.

20 (a) D. Ding, C. C. Goh, G. Feng, Z. Zhao, J. Liu, R. Liu, N. Tomczak, J. Geng, B. Z. Tang, L. G. Ng and B. Liu,
Ultrabright organic dots with aggregation-induced emission characteristics for real-time two-photon intravital vasculature imaging, Adv. Mater., 2013, 25, 6083-6088; (b) D. Cheng, Y. Pan, L. Wang, Z. Zeng, L. Yuan, X. Zhang and Y. T. Chang, Selective visualization of the endogenous peroxynitrite in an inflamed mouse model by a mitochondria-targetable twophoton ratiometric fluorescent probe, J. Am. Chem. Soc., 2017, 139, 285-292; (c) S. Zhen, S. Wang, S. Li, W. Luo, M. Gao, L. G. Ng, C. C. Goh, A. Qin, Z. Zhao, B. Liu and B. Z. Tang, Efficient red/near-infrared fluorophores based on benzo[1,2-b: 4,5- $\left.\mathrm{b}^{\prime}\right]$ dithiophene 1,1,5,5-tetraoxide for targeted photodynamic therapy and in vivo two-photon fluorescence bioimaging, $A d v$. Funct. Mater., 2018, 28, 1706945.

21 Y. Nakamura, A. Mochida, P. L. Choyke and H. Kobayashi, Nanodrug delivery: is the enhanced permeability and retention effect sufficient for curing cancer?, Bioconjugate Chem., 2016, 27, 2225-2238. 